\title{
Effect of the deformation parameter on the nonrelativistic energy spectra of the q-deformed Hulthen-quadratic exponential-type potential
}

Ushie Patrick Obogo ${ }^{1} \oplus$, Ofem Egbe Ubi ${ }^{1} \oplus$, Collins Okon Edet ${ }^{2+} \oplus$, Akpan Ndem $\operatorname{Ikot}^{2}{ }^{\circledR}$

1. Cross River University of Technology, Department of Physics, Faculty of Physical Sciences, Calabar, Nigeria.

2. University of Port Harcourt, Department of Physics, Rivers State, Nigeria.

+Corresponding author: Collins Okon Edet, Phone: +2349016715883, Email address: collinsokonedet@gmail.com

\section{ARTICLE INFO}

Article history:

Received: October 20, 2020

Accepted: May 27, 2021

Published: October 01, 2021

\section{Keywords}

1. Schrödinger equation

2. potential model

3. bound state

4. parametric Nikiforov-Uvarov (NU) method

Section Editor: Assis Vicente Benedetti

ABSTRACT: In this study, an approximate solution of the Schrödinger equation for the $q$ deformed Hulthen-quadratic exponential-type potential model within the framework of the Nikiforov-Uvarov method was obtained. The bound state energy equation and the corresponding eigenfunction was obtained. The energy spectrum is applied to study $\mathrm{H}_{2}, \mathrm{HCl}$, $\mathrm{CO}$ and $\mathrm{LiH}$ diatomic molecules. The effect of the deformation parameters and other potential parameters on the energy spectra of the system were graphically and numerically analyzed in detail. Special cases were considered when the
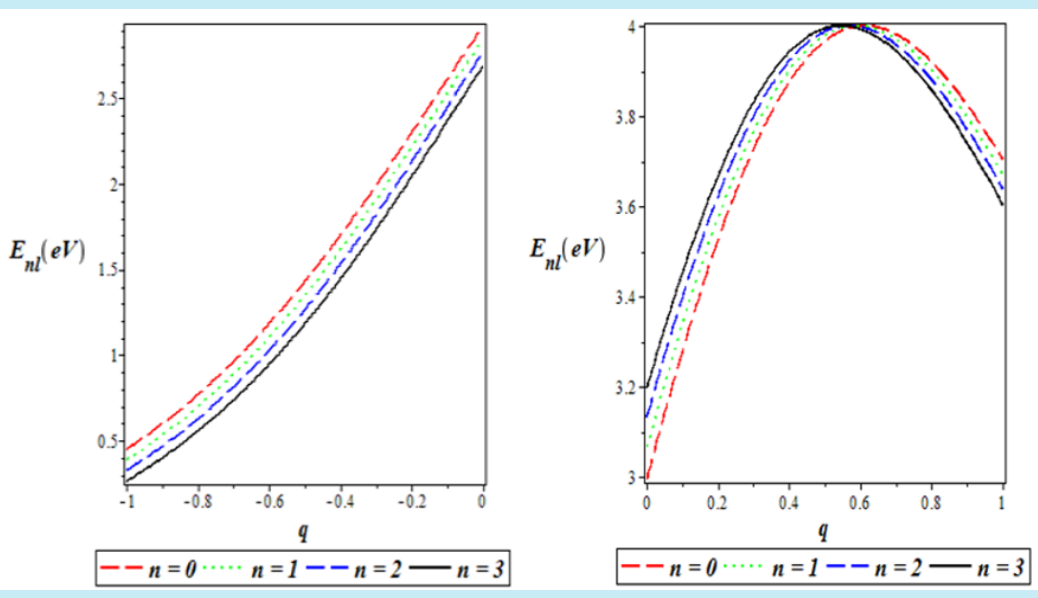
potential parameters were altered, resulting in deformed Hulthen potential, Hulthen potential, deformed quadratic exponential-type potential and quadratic exponential-type potential. The energy eigenvalues expressions agreed with what obtained in literature. Finally, the results can find many applications in quantum chemistry, atomic and molecular physics. 


\section{Introduction}

Since the early days of quantum mechanics (QM), the study of a particle confined by a potential field has been of utmost importance (Edet and Okoi, 2019; Edet et al., 2020a; b; Landau, 1977; Schiff, 1995). Studies of this nature are carried out by solving the Schrödinger equation with different interaction potentials of interest. The solutions of the Schrödinger equation with different potential models of interest have been employed by many researchers to give insights, explanations, and predictions into the behavior of diatomic molecules, quarks, etc (Edet et al., 2020c; 2021a; Greiner, 2000; Okoi et al., 2020; Okorie et al., 2019).

Recently, numerous researchers have proffered solutions to the Schrödinger equation for some potential of interest. The analytical solution of the Schrödinger equation with $\ell=0$ and $\ell \neq 0$ for some potentials has been addressed by many researchers in non-relativistic and relativistic quantum mechanics for bound states (Durmus and Yasuk, 2007; Edet et al., 2020d; 2021b; Louis et al., 2018a; 2018b). Some of these potentials include Deng-Fan potential (Falaye et al., 2015), Hyperbolic potential (Onate et al., 2018b), Eckart potential (Onate et al., 2017), generalized trigonometric Pöschl-Teller potential (Edet et al., 2020e), and screened Kratzer Potential (Ikot et al., 2020a).

Also, several methods have been employed to obtain the solutions of the nonrelativistic wave equations with some potential models of interest, some of these methods include: the factorization method (Dong, 2007), formula method (Falaye et al., 2015), supersymmetry quantum mechanics (SUSYQM) (Falaye et al., 2014), Nikiforov-Uvarov method (NU) (Nikiforov and Uvarov, 1988) asymptotic iteration method (AIM) (Ciftci et al., 2003; 2005; Falaye, 2012), exact quantization Rule (Gu and Dong, 2011; Ma and $\mathrm{Xu}, 2005$ ), proper quantization rule (Qiang and Dong, 2007), WKBJ (Ita et al., 2018), etc.

Moreover, it is the goal in the present consideration to propose a potential of the form:
$V_{q}(r)=\frac{V_{0} e^{-\alpha r}}{1-q e^{-\alpha r}}+\frac{V_{1}\left(a+b e^{-\alpha r}+c e^{-2 \alpha r}\right)}{\left(1-q e^{-\alpha r}\right)^{2}}$

Where $V_{0}$ and $V_{l}$ are the potential strengths, $\alpha$ is the screening parameter, $a, b$ and $c$ are the adjusted parameter and $q$ is deformation parameter. We call the potential $q$-deformed Hulthen-quadratic exponentialtype potential ( $q$-HQEP). With respect to what was obtained in previous studies of the molecular potential, the potential to allow for more physical application and a comparative analysis to existing studies of the molecular potential was modified. In addition, in molecular physics, it has also been established that potential energy functions with more parameters tend to fit experimental data than those with fewer parameters and researchers have recently paid great attention to obtaining modified version of potential functions by employing dissociation energy, and equilibrium bond length for molecular systems as explicit parameters. This model will be an important tool for spectroscopists to represent experimental data, verify measurements, and make predictions.

The potential is a superposition of the Hulthen (Ikhdair, 2009; Ikhdair and Sever, 2007; Onate et al., 2018a) and Quadratic exponential-type potentials (Okorie et al., 2018). These potentials have been individually applied to carry out studies extensively by several researchers (Ikot et al., 2014). Hence, the motivation to combine them.

In this research article, the goal is in two-fold. First, the Schrödinger wave equation (SWE) is solved with the $q$-HQEP via parametric Nikiforov-Uvarov method. The effect of the deformation parameter on the energy spectra of some diatomic molecules is analyzed with the aid of some graphical representation and numerical analysis.

The outline of the paper is as follows: section 2 provides brief a description of the parametric Nikiforov-Uvarov method. In section 3, the solutions of the three-dimensional (3D) Schrödinger equation (SE) with the $q$-HQEP via parametric NU. In Section 4 , special cases of the potential understudy were discussed. In section 5, the results of this study are presented and discussed. Finally, in section 6, the concluding remarks are given.

\section{The parametric NU method}

The parametric form of the NU method takes the form (Tezcan and Sever, 2009):

$\frac{d^{2} \psi}{d s^{2}}+\frac{\alpha_{1}-\alpha_{2} s}{s\left(1-\alpha_{3} s\right)} \frac{d \psi}{d s}+\frac{1}{s^{2}\left(1-\alpha_{3} s\right)^{2}}\left\{-\xi_{1} s^{2}+\xi_{2} s-\xi_{3}\right\} \psi(s)=0$ 
The energy eigenvalues equation and eigenfunctions satisfy the following sets of equations, respectively:

$\alpha_{2} n-(2 n+1) \alpha_{5}+(2 n+1)\left(\sqrt{\alpha_{9}}+\alpha_{3} \sqrt{\alpha_{8}}\right)+n(n-1) \alpha_{3}+\alpha_{7}+2 \alpha_{3} \alpha_{8}+2 \sqrt{\alpha_{8} \alpha_{9}}=0$

$\psi(s)=s^{\alpha_{12}}\left(1-\alpha_{3} s\right)^{-\alpha_{12}-\frac{\alpha_{13}}{\alpha_{3}}} P_{n}^{\left(\alpha_{10}-1, \frac{\alpha_{11}}{\alpha_{3}}-\alpha_{10}-1\right)}\left(1-2 \alpha_{3} s\right)$

where

$\alpha_{4}=\frac{1}{2}\left(1-\alpha_{1}\right), \alpha_{5}=\frac{1}{2}\left(\alpha_{2}-2 \alpha_{3}\right), \alpha_{6}=\alpha_{5}^{2}+\xi_{1}$

$\alpha_{7}=2 \alpha_{4} \alpha_{5}-\xi_{2}, \alpha_{8}=\alpha_{4}^{2}+\xi_{3}, \alpha_{9}=\alpha_{3} \alpha_{7}+\alpha_{3}^{2} \alpha_{8}+\alpha_{6}$

$\alpha_{10}=\alpha_{1}+2 \alpha_{4}+2 \sqrt{\alpha_{8}}, \alpha_{11}=\alpha_{2}-2 \alpha_{5}+2\left(\sqrt{\alpha_{9}}+\alpha_{3} \sqrt{\alpha_{8}}\right)$

$\alpha_{12}=\alpha_{4}+\sqrt{\alpha_{8}}, \alpha_{13}=\alpha_{5}-\left(\sqrt{\alpha_{9}}+\alpha_{3} \sqrt{\alpha_{8}}\right)$

and $P_{n}$ is the orthogonal Jacobi polynomial, which is defined as

$P_{n}^{(\alpha, \beta)}(x)=\frac{\Gamma(\alpha+n+1)}{n ! \Gamma(\alpha+\beta+n+1)} \sum_{m=0}^{n}\left(\begin{array}{l}n \\ m\end{array}\right) \frac{\Gamma(\alpha+\beta+n+m+1)}{\Gamma(\alpha+m+1)}\left(\frac{x-1}{2}\right)^{m}$

\section{Bound-state solutions of $q$-deformed Hulthen plus quadratic exponential-type potential}

The radial Schrödinger equation in arbitrary dimensions (Rampho et al., 2021; Ebomwonyi et al., 2017) can be given as:

$\frac{d^{2} R_{n \ell}(r)}{d r^{2}}+\frac{2 \mu}{\hbar^{2}}\left[E_{n \ell}-V(r)-\frac{\ell(\ell+1) \hbar^{2}}{2 \mu r^{2}}\right] R_{n \ell}(r)=0$

where $\mu$ is the reduced mass, $E_{n \ell}$ is the energy spectrum, $\hbar$ is the reduced Planck's constant and $n$ and $\ell$ are the principal and orbital angular momentum quantum numbers, respectively (or vibration-rotation quantum numbers in quantum chemistry) (Onate et al., 2018c). Substituting Eq. 1 into Eq. 7 gives:

$\frac{d^{2} R_{n \ell}(r)}{d r^{2}}+\left[\frac{2 \mu E_{n \ell}}{\hbar^{2}}-\frac{2 \mu}{\hbar^{2}}\left(-\frac{V_{0} e^{-\alpha r}}{1-q e^{-\alpha r}}+\frac{V_{1}\left(a+b e^{-\alpha r}+c e^{-2 \alpha r}\right)}{\left(1-q e^{-\alpha r}\right)^{2}}\right)-\frac{\ell(\ell+1)}{r^{2}}\right] R_{n \ell}(r)=0$

The radial Schrödinger equation with this potential can be solved exactly for $\ell=0$ (s-wave) but cannot be solved with this potential for $\ell \neq 0$. To obtain the solution for $\ell \neq 0$, the approximation scheme proposed by Greene and Aldrich (1976) is employed to deal with the centrifugal term, which is given as:

$\frac{1}{r^{2}} \approx \frac{\alpha^{2} e^{-\alpha r}}{\left(1-q e^{-\alpha r}\right)^{2}}$

It is noted that for a short-range potential, the relation in Eq. 9 is a good approximation to $\frac{1}{r^{2}}$, as proposed by Greene and Aldrich (1976), Ikot et al. (2020b) and Okorie et al. (2020). This implies that Eq. 9 is not a good approximation to the centrifugal barrier when the screening parameter $\alpha$ becomes large. Thus, the approximation is valid when $\alpha \ll 1$. Substituting the approximation Eq. 8 into Eq. 9, an equation of the form is obtained:

$\frac{d^{2} R_{n \ell}(r)}{d r^{2}}+\left[\frac{2 \mu E_{n \ell}}{\hbar^{2}}-\frac{2 \mu}{\hbar^{2}}\left(\frac{V_{0} e^{-\alpha r}}{1-q e^{-\alpha r}}+\frac{V_{1}\left(a+b e^{-\alpha r}+c e^{-2 \alpha r}\right)}{\left(1-q e^{-\alpha r}\right)^{2}}\right)-\frac{\ell(\ell+1) \alpha^{2} e^{-2 \alpha r}}{\left(1-q e^{-\alpha r}\right)^{2}}\right] R_{n \ell}(r)=0$

To solve the differential equation above, the transformation $s=e^{-\alpha r}$ is used so as to enable to apply the NU method as a solution technique to the hypergeometric-type differential equation. Hence, this transforms $\frac{d^{2} R_{n \ell}(r)}{d r^{2}}$ into the form: 
$\frac{d^{2} R_{n \ell}(r)}{d r^{2}}=\alpha^{2} s^{2} \frac{d^{2} R_{n \ell}(s)}{d s^{2}}+\alpha^{2} s \frac{d R_{n \ell}(s)}{d s}$

$\alpha^{2} s^{2} \frac{d^{2} R_{n \ell}(s)}{d s^{2}}+\alpha^{2} s \frac{d R_{n \ell}(s)}{d s}+\left[\begin{array}{l}\frac{2 \mu E_{n \ell}}{\hbar^{2}}-\frac{2 \mu}{\hbar^{2}}\left(\frac{V_{0} s}{1-q s}+\frac{V_{1}\left(a+b s+c s^{2}\right)}{(1-q s)^{2}}\right) \\ -\frac{\ell(\ell+1) \alpha^{2} s}{(1-q s)^{2}}\end{array}\right] R_{n \ell}(s)=0$

In view of the above, the differential equation of the form is obtained:

$\frac{d^{2} R_{n \ell}(s)}{d s^{2}}+\frac{1-q s}{s(1-q s)} \frac{d R_{n \ell}(s)}{d s}+\frac{1}{s^{2}(1-q s)^{2}}\left[\begin{array}{l}-\left(\varepsilon_{n \ell} q^{2}+\beta q-\delta_{3}\right) s^{2}+ \\ \left(2 \varepsilon_{n \ell} q+\beta-\delta_{3}-\gamma\right) s-\left(\varepsilon_{n \ell}-\delta_{3}\right)\end{array}\right] R_{n \ell}(s)=0$

where the following dimensionless abbreviations have been introduced for mathematical convenience:

$-\varepsilon_{n \ell}=\frac{2 \mu E_{n \ell}}{\hbar^{2} \alpha^{2}}, \beta=\frac{2 \mu V_{0}}{\hbar^{2} \alpha^{2}}, \delta_{1}=\frac{2 \mu V_{1} a}{\hbar^{2} \alpha^{2}}, \delta_{2}=\frac{2 \mu V_{1} b}{\hbar^{2} \alpha^{2}}, \delta_{3}=\frac{2 \mu V_{1} c}{\hbar^{2} \alpha^{2}} \gamma=\ell(\ell+1)$

Equation 13 is of the form that is solvable by the NU method. Therefore, on comparison to Eqs. 2 and 13 , the following parameters are obtained:

$\xi_{1}=\varepsilon_{n \ell} q^{2}+\beta q-\delta_{3}$

$\xi_{2}=2 \varepsilon_{n \ell} q+\beta-\delta_{3}-\gamma$

$\xi_{3}=\varepsilon_{n \ell}-\delta_{3}$

and

$\alpha_{1}=1, \alpha_{2}=q, \alpha_{3}=q$

$\xi_{1}=\varepsilon_{n \ell} q^{2}+\beta q-\delta_{3}, \xi_{2}=2 \varepsilon_{n \ell} q+\beta-\delta_{3}-\gamma, \xi_{3}=\varepsilon_{n \ell}-\delta_{3}$

$\alpha_{4}=0, \alpha_{5}=-\frac{q}{2}, \alpha_{6}=\frac{q^{2}}{4}+\varepsilon_{n \ell} q^{2}+\beta q-\delta_{3}, \alpha_{7}=-2 \varepsilon_{n \ell} q-\beta+\delta_{3}+\gamma$

$\alpha_{8}=\varepsilon_{n \ell}+\delta_{1}, \alpha_{9}=\frac{q^{2}}{4}-\delta_{2} q+\gamma q+\delta_{1} q^{2}-\delta_{3}$

$\alpha_{10}=1+2 \sqrt{\varepsilon_{n \ell}-\delta_{1}}, \alpha_{11}=2 q+2\left(\sqrt{\frac{q^{2}}{4}-\delta_{2} q+\gamma q+\delta_{1} q^{2}-\delta_{3}}+q \sqrt{\varepsilon_{n \ell}+\delta_{1}}\right)$

$\alpha_{12}=\sqrt{\varepsilon_{n \ell}+\delta_{1}}, \alpha_{13}=-\frac{q}{2}-\left(\sqrt{\frac{q^{2}}{4}-\delta_{2} q+\gamma q+\delta_{1} q^{2}-\delta_{3}}+q \sqrt{\varepsilon_{n \ell}+\delta_{1}}\right)$

Substituting these polynomials into Eq. 3, it is possible to obtain

$q\left(n+\frac{1}{2}\right)^{2}+2\left(n+\frac{1}{2}\right)\left(\sqrt{\frac{q^{2}}{4}-\delta_{2} q+\gamma q+\delta_{1} q^{2}-\delta_{3}}+q \sqrt{\varepsilon_{n \ell}+\delta_{1}}\right)-\beta+\delta_{2}+\gamma+2 \delta_{1} q+\frac{q}{4}+$

$2 \sqrt{\left(\varepsilon_{n \ell}+\delta_{1}\right)\left(\frac{q^{2}}{4}-\delta_{2} q+\gamma q+\delta_{1} q^{2}-\delta_{3}\right)}=0$

By carrying out some algebraic manipulation, the following equation is obtained:

$\varepsilon_{n \ell}=-\delta_{1}+\frac{1}{4}\left[\frac{\left(n+\frac{1}{2}+\sqrt{\frac{1}{4}-\frac{\delta_{2}}{q}+\frac{\gamma}{q}+\delta_{1}-\frac{\delta_{3}}{q^{2}}}\right)^{2}+\frac{\delta_{3}}{q^{2}}+\delta_{1}-\frac{\beta}{q}}{\left(n+\frac{1}{2}+\sqrt{\frac{1}{4}-\frac{\delta_{2}}{q}+\frac{\gamma}{q}+\delta_{1}-\frac{\delta_{3}}{q^{2}}}\right)}\right]^{2}$

Substituting Eq. 14 into Eq. 17 and carrying some simple manipulative algebra, it is possible to arrive at the energy eigenvalue equation of the $q$-deformed Hulthen plus quadratic exponential-type potential in the form: 
$E_{n \ell}=V_{1} a-\frac{\hbar^{2} \alpha^{2}}{8 \mu}\left[\begin{array}{l}\left.\left(n+\frac{1}{2}+\sqrt{\frac{1}{4}-\frac{2 \mu V_{1} b}{\hbar^{2} \alpha^{2} q}+\frac{\ell(\ell+1)}{q}+\frac{2 \mu V_{1} a}{\hbar^{2} \alpha^{2}}-\frac{2 \mu V_{1} c}{\hbar^{2} \alpha^{2} q^{2}}}\right)^{2}+\right]^{\frac{2 \mu V_{1} c}{\hbar^{2} \alpha^{2} q^{2}}+\frac{2 \mu V_{1} a}{\hbar^{2} \alpha^{2}}-\frac{2 \mu V_{0}}{\hbar^{2} \alpha^{2} q}} \\ \left(n+\frac{1}{2}+\sqrt{\frac{1}{4}-\frac{2 \mu V_{1} b}{\hbar^{2} \alpha^{2} q}+\frac{\ell(\ell+1)}{q}+\frac{2 \mu V_{1} a}{\hbar^{2} \alpha^{2}}-\frac{2 \mu V_{1} c}{\hbar^{2} \alpha^{2} q^{2}}}\right)\end{array}\right]^{2}$

The corresponding wave functions can be evaluated from Eq. 4 as follows:

$R_{n \ell}(s)=N_{n \ell} S^{\sqrt{\varepsilon_{n \ell}+\delta_{1}}}(1-s)^{\frac{1}{2}+\sqrt{\frac{1}{4}-\frac{\delta_{2}}{q}+\frac{\gamma}{q}+\delta_{1}-\frac{\delta_{3}}{q^{2}}}} P_{n}^{\left(2 \sqrt{\varepsilon_{n \ell}+\delta_{1}}, 2 \sqrt{\frac{1}{4}-\frac{\delta_{2}}{q}+\frac{\gamma}{q}+\delta_{1}-\frac{\delta_{3}}{q^{2}}}\right)}(1-2 s)$

From the definition of the Jacobi polynomials (Edet et al., 2020a; b).

$P_{n}^{(2 \varpi, 2 \chi)}(1-2 s)=\frac{\Gamma(n+2 \varpi+1)}{n ! \Gamma(2 \varpi+1)}{ }_{2} F_{1}(-n, 2 \varpi+2 \chi+n+1,2 \varpi+1 ; s)$

$\varpi=\sqrt{\varepsilon_{n \ell}+\delta_{1}}$

$\chi=\sqrt{\frac{1}{4}-\frac{\delta_{2}}{q}+\frac{\gamma}{q}+\delta_{1}-\frac{\delta_{3}}{q^{2}}}$

In terms of hypergeometric polynomials, Eq. 21 can be written as

$R_{n \ell}(s)=N_{n \ell} s^{\varpi}(1-s)^{\frac{1}{2}+\chi} \frac{\Gamma(n+2 \varpi+1)}{n ! \Gamma(2 \varpi+1)}{ }_{2} F_{1}(-n, 2 \varpi+2 \chi+n+1,2 \varpi+1 ; s)$

\section{Special cases}

\subsection{Deformed Hulthen potential}

If $V_{1}$ is set as $V_{1}=a=b=c=0$, the potential model (1) reduces to deformed Hulthen potential (Hall et al., 2018):

$V_{q}(r)=\frac{V_{0} e^{-\alpha r}}{1-q e^{-\alpha r}}$

and the energy equation as follows:

$E_{n \ell}=-\frac{\hbar^{2} \alpha^{2}}{8 \mu}\left[\frac{\left(n+\frac{1}{2}+\sqrt{\frac{1}{4}+\frac{\ell(\ell+1)}{q}}\right)^{2}-\frac{2 \mu V_{0}}{\hbar^{2} \alpha^{2} q}}{\left(n+\frac{1}{2}+\sqrt{\frac{1}{4}+\frac{\ell(\ell+1)}{q}}\right)}\right]^{2}$

This is in agreement with Eq. 44 (Edet and Okoi, 2019).

\subsection{Hulthen potential}

If $V_{1}$ is set as $V_{1}=a=b=c=0$ and $q \rightarrow 1$, the potential model (1) reduces to the Hulthen potential: 
$V(r)=\frac{V_{0} e^{-\alpha r}}{1-e^{-\alpha r}}$

and the energy equation as follows:

$E_{n \ell}=-\frac{\hbar^{2} \alpha^{2}}{8 \mu}\left[\frac{\left(n+\frac{1}{2}+\sqrt{\frac{1}{4}+\ell(\ell+1)}\right)^{2}-\frac{2 \mu V_{0}}{\hbar^{2} \alpha^{2}}}{\left(n+\frac{1}{2}+\sqrt{\frac{1}{4}+\ell(\ell+1)}\right)}\right]^{2}$

Equation 26 is in agreement with the energy equation given in Eq. 39 (Ikhdair, 2009), Eq. 31 (Ikhdair and Sever, 2007), Eq. 34 (Agboola, 2009), Eq. 35 (Bayrak et al., 2006) and Eq. 27 (Jia et al., 2008).

\subsection{Deformed quadratic exponential-type potential}

If $V_{0}$ is set as $V_{0}=0$, the potential model (1) reduces to deformed quadratic exponential-type potential:

$V_{q}(r)=\frac{V_{1}\left(a+b e^{-\alpha r}+c e^{-2 \alpha r}\right)}{\left(1-q e^{-\alpha r}\right)^{2}}$

and the energy equation as follows:

$E_{n \ell}=V_{1} a-\frac{\hbar^{2} \alpha^{2}}{8 \mu}\left[\begin{array}{l}\left.\left(n+\frac{1}{2}+\sqrt{\frac{1}{4}-\frac{2 \mu V_{1} b}{\hbar^{2} \alpha^{2} q}+\frac{\ell(\ell+1)}{q}+\frac{2 \mu V_{1} a}{\hbar^{2} \alpha^{2}}-\frac{2 \mu V_{1} c}{\hbar^{2} \alpha^{2} q^{2}}}\right)^{2}+\right]^{2}+\frac{2 \mu V_{1} a}{\hbar^{2} \alpha^{2}} \\ \left(n+\frac{1}{2}+\sqrt{\frac{1}{4}-\frac{2 \mu V_{1} b}{\hbar^{2} \alpha^{2} q}+\frac{\ell(\ell+1)}{q}+\frac{2 \mu V_{1} a}{\hbar^{2} \alpha^{2}}-\frac{2 \mu V_{1} c}{\hbar^{2} \alpha^{2} q^{2}}}\right)\end{array}\right]^{2}$

\subsection{Quadratic exponential-type potential}

If $V_{0}$ is set as $V_{0}=0$ and $q \rightarrow 1$ the potential model (1) reduces to quadratic exponential-type potential (Okorie et al., 2018):

$V(r)=\frac{V_{1}\left(a+b e^{-\alpha r}+c e^{-2 \alpha r}\right)}{\left(1-e^{-\alpha r}\right)^{2}}$

and the energy equation as follows:

$E_{n \ell}=V_{1} a-\frac{\hbar^{2} \alpha^{2}}{8 \mu}\left[\begin{array}{l}\left(n+\frac{1}{2}+\sqrt{\frac{1}{4}-\frac{2 \mu V_{1} b}{\hbar^{2} \alpha^{2}}+\ell(\ell+1)+\frac{2 \mu V_{1} a}{\hbar^{2} \alpha^{2}}-\frac{2 \mu V_{1} c}{\hbar^{2} \alpha^{2}}}\right)^{2}+ \\ \frac{2 \mu V_{1} c}{\hbar^{2} \alpha^{2}}+\frac{2 \mu V_{1} a}{\hbar^{2} \alpha^{2}} \\ \left(n+\frac{1}{2}+\sqrt{\frac{1}{4}-\frac{2 \mu V_{1} b}{\hbar^{2} \alpha^{2}}+\ell(\ell+1)+\frac{2 \mu V_{1} a}{\hbar^{2} \alpha^{2}}-\frac{2 \mu V_{1} c}{\hbar^{2} \alpha^{2}}}\right)\end{array}\right]^{2}$

Equation 30 is in agreement with the energy equation given in Eq. 31 (Okorie et al., 2018). 


\section{Results and discussions}

In our study, the energy (Eq. 18) and wave function (Eq. 22) of the deformed Hulthen potential plus the quadratic exponential-type potential obtained using the parametric NU. For validity purposes, were also obtained the energy eigenvalues and wave function of the deformed Hulthen potential, Hulthen potential, deformed quadratic exponential-type potential and quadratic exponential-type potential, shown in Eqs. 23-30 as special cases.
In the present study, the energy spectrum was used to study the four selected diatomic molecules, $\mathrm{H}_{2}, \mathrm{HCl}$, $\mathrm{CO}$ and $\mathrm{LiH}$. The spectroscopic parameters of these molecules are given in Tab. 1 and taken from (Edet et al., 2020e; Rampho et al., 2021). The following conversions were used; $\hbar \mathrm{c}=1973.269 \mathrm{eVA}$ and $1 \mathrm{amu}=931.5 \times 10^{6} \mathrm{eV}(\AA)^{-1}$ for all computations (Edet et al., 2020e; Rampho et al., 2021). All nonspectroscopic parameters are kept in natural units.

Table 1. Spectroscopic parameters of the molecules used in this work (Edet et al., 2020e; Rampho et al., 2021).

\begin{tabular}{|c|c|c|c|c|}
\hline Parameters & $\begin{array}{c}\mathrm{H}_{2} \\
\text { (Edet et al., 2020e) }\end{array}$ & $\begin{array}{c}\text { HCl } \\
\text { (Rampho et al., 2021) }\end{array}$ & $\begin{array}{c}\text { CO } \\
\text { (Edet et al., 2020e) }\end{array}$ & $\begin{array}{c}\text { LiH } \\
\text { (Rampho et al., 2021) }\end{array}$ \\
\hline$\alpha\left(\AA^{-1}\right)$ & 1.9426 & 1.8677 & 2.294 & 1.128 \\
\hline$\mu(a . m . u)$ & 0.50391 & 0.980105 & 6.860672 & 0.880122 \\
\hline
\end{tabular}

In Tab. 2, the energy values for the $q$-deformed Hulthen-quadratic exponential-type potential was shown for $\mathrm{H}_{2}, \mathrm{HCl}, \mathrm{CO}$ and $\mathrm{LiH}$ diatomic molecules for various values of the deformation parameter and of quantum states. It is seen clearly that when the deformation parameter is $\mathrm{q}=-0.5$, the energy is low and for instance in $\mathrm{LiH}$ molecule it becomes more bounded. But for $\mathrm{q}=0.5$, the energy is raised significantly although drops slightly and remains positive when $\mathrm{q}=1$ (i.e., absence of deformation).

Table 2. Energy values for the $q$-deformed Hulthen-quadratic exponential-type potential for $\mathrm{H}_{2}, \mathrm{HCl}, \mathrm{CO}$ and $\mathrm{LiH}$ diatomic molecule for various values of the deformation parameter and of quantum states.

\begin{tabular}{|c|c|c|c|c|c|}
\hline State & $q$ & $\mathrm{H}_{2}$ & $\mathrm{HCl}$ & $\mathrm{CO}$ & $\mathrm{LiH}$ \\
\hline \multirow{3}{*}{$1 \mathrm{~s}$} & -0.5 & 1.208540 & 1.104410 & 2.021420 & -0.338560 \\
\hline & 0.5 & 3.865880 & 3.710050 & 4.565230 & 2.225200 \\
\hline & 1.0 & 3.547310 & 3.415690 & 4.299980 & 1.950540 \\
\hline \multirow{3}{*}{$2 \mathrm{~s}$} & -0.5 & 0.910264 & 0.901356 & 1.928360 & -0.466642 \\
\hline & 0.5 & 3.885060 & 3.730360 & 4.578700 & 2.241860 \\
\hline & 1.0 & 3.423210 & 3.333520 & 4.263660 & 1.899950 \\
\hline \multirow{3}{*}{$2 p$} & -0.5 & 1.221470 & 1.110510 & 2.022730 & -0.336094 \\
\hline & 0.5 & 3.867670 & 3.711040 & 4.565480 & 2.225650 \\
\hline & 1.0 & 3.542160 & 3.413300 & 4.299470 & 1.949580 \\
\hline \multirow{3}{*}{$3 \mathrm{~s}$} & -0.5 & 0.600211 & 0.692631 & 1.834060 & -0.597053 \\
\hline & 0.5 & 3.872680 & 3.735150 & 4.588670 & 2.252040 \\
\hline & 1.0 & 3.284190 & 3.243990 & 4.225680 & 1.846270 \\
\hline \multirow{3}{*}{$3 p$} & -0.5 & 0.923715 & 0.907634 & 1.929690 & -0.464130 \\
\hline & 0.5 & 3.885170 & 3.730790 & 4.578890 & 2.242160 \\
\hline & 1.0 & 3.417380 & 3.330900 & 4.263140 & 1.898930 \\
\hline \multirow{3}{*}{$3 d$} & -0.5 & 1.247280 & 1.122710 & 2.025350 & -0.331163 \\
\hline & 0.5 & 3.870960 & 3.712950 & 4.565970 & 2.226530 \\
\hline & 1.0 & 3.531790 & 3.408500 & 4.298470 & 1.947680 \\
\hline \multirow{3}{*}{$4 \mathrm{~s}$} & -0.5 & 0.278707 & 0.478346 & 1.738530 & -0.729764 \\
\hline & 0.5 & 3.830940 & 3.725190 & 4.595210 & 2.255940 \\
\hline & 1.0 & 3.131300 & 3.147490 & 4.186070 & 1.789610 \\
\hline \multirow{3}{*}{$4 p$} & -0.5 & 0.614176 & 0.699079 & 1.835410 & -0.594496 \\
\hline & 0.5 & 3.871240 & 3.735050 & 4.588800 & 2.252200 \\
\hline & 1.0 & 3.277720 & 3.241150 & 4.225130 & 1.845200 \\
\hline
\end{tabular}




\begin{tabular}{|c|c|c|c|c|c|c|}
\hline \multirow{3}{*}{$4 \mathrm{~d}$} & -0.5 & 0.950587 & 0.920182 & 1.932350 & -0.459108 \\
\cline { 2 - 7 } & 0.5 & 3.885150 & 3.731590 & 4.579270 & 2.242750 \\
\hline \multirow{3}{*}{$4 \mathrm{f}$} & 1.0 & 3.405660 & 3.325650 & 4.262080 & 1.896900 \\
\cline { 2 - 7 } & -0.5 & 1.285910 & 1.140990 & 2.029280 & -0.323769 \\
\hline
\end{tabular}

The variation of the energy eigenvalues with different parameters of the combined potential, such as $V_{0}, V_{l}, a, b, c$ and $q$, is shown in Figs. 1-7, respectively, for $\mathrm{H}_{2}, \mathrm{HCl}, \mathrm{CO}$ and $\mathrm{LiH}$ diatomic molecule in the ground state. In Fig. $1 \mathrm{a}$ and b, the variation of the energy spectrum was plotted for various values of $n$ as a function of the parameter $\ell$ for $\mathrm{q}=1$ and $\mathrm{q}=-1$, respectively. Figure 1 a shows that the energy decreases as the angular momentum increases. In Fig. 1b, it is possible to observe that the energy increases as the $\ell$ increases up to a maximum and then drops again sporadically. Figure $2 \mathrm{a}$ and $\mathrm{b}$ shows the variation of the energy spectrum for various values of $n$ as a function of the parameter $V_{0}$ for $\mathrm{q}=1$ and $q=1$, respectively, in the region $0<V_{0}<3$. In Fig. 2a, the energy of the system increases as the parameter $V_{0}$ increases. In Fig. $2 \mathrm{~b}$, the energy linearly decreases as $V_{0}$ increases. Figure $3 \mathrm{a}$ and $\mathrm{b}$ shows the variation of the energy spectrum for various values of $n$ as a function of the parameter $V_{l}$ for $\mathrm{q}=1$ and $\mathrm{q}=$ -1 , respectively, in the region $0<V_{1}<0.16$. In both figures, the energy increases monotonically as the parameter $V_{l}$ increases as well. Figure $4 \mathrm{a}$ and b shows the variation of the energy spectrum for various values of diatomic molecules as a function of the parameter $a$, respectively, in the interval $0<\mathrm{a}<0.25$. In both cases considered, the energy increases linearly with increasing $a$, but in the case $q=-1$ (Fig. 4b), there is a wider spacing between the energy levels. Figure $5 \mathrm{a}$ and $b$ shows the variation of the energy spectrum for various values of $n$ as a function of the parameter $b$ for $\mathrm{q}=1$ and $\mathrm{q}=-1$, respectively, in the region $0<\mathrm{b}<$ 1. In Fig. 5a, the energy increases linearly as the parameter $b$ increases. In Fig. 5b, the energy decreases linearly as the parameter $b$ increases. Figure $6 a$ and $b$ shows the variation of the energy spectrum for various values of $n$ as a function of the parameter $c$ for $\mathrm{q}=1$ and $\mathrm{q}=-1$, respectively, in the region $0<\mathrm{c}<0.20$. In Fig. 6a, the energy decreases linearly as the parameter $c$ increases. In Fig. 6b, the energy decreases linearly as the parameter $c$ increases. Figure $7 \mathrm{a}$ and $\mathrm{b}$ shows the variation of the energy spectrum for various values of $n$ as a function of the parameter $-q$ and $+q$ in the intervals $-1<q<0$ and $0<q<1$, respectively. In Fig. 7a, the energy increases up to a maximum as $q$ increases and then declines again. In Fig. 7b, the energy increases linearly as $q$ upsurges.
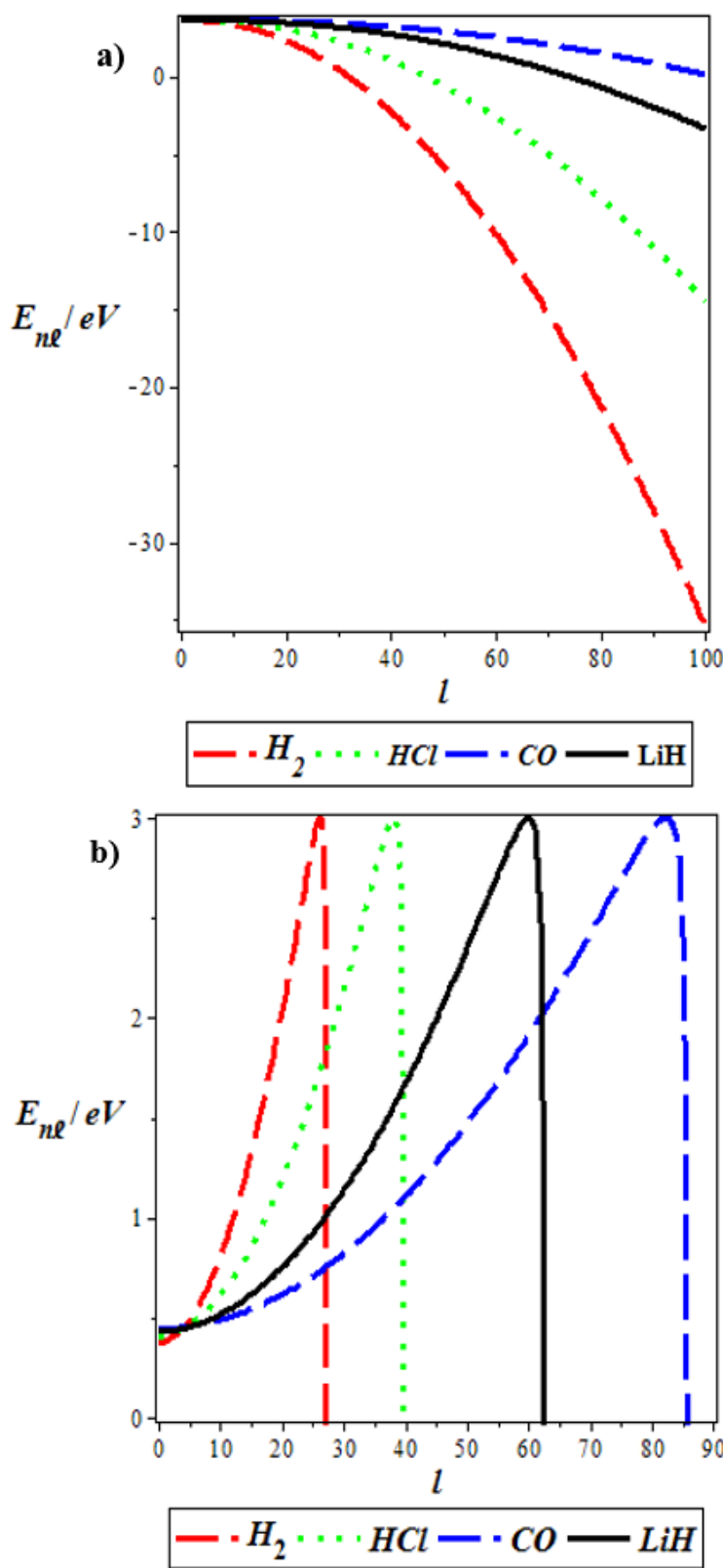

Figure 1. (a) The variation of the energy spectrum for various values of $n$ as a function of the parameter $\ell$ for $q=1$. (b) The variation of the energy spectrum for various values of $n$ as a function of the parameter $\ell$ for $\mathrm{q}=-1 . \quad \mathrm{a}=2 \mathrm{fm}^{-1}, \mathrm{~b}=1 \mathrm{fm}^{-1}, \mathrm{c}=-3 \mathrm{fm}^{-1}, \mathrm{~V}_{1}=$ $2 \mathrm{fm}^{-1}$ and $V_{0}=3 \mathrm{fm}^{-1}$. 

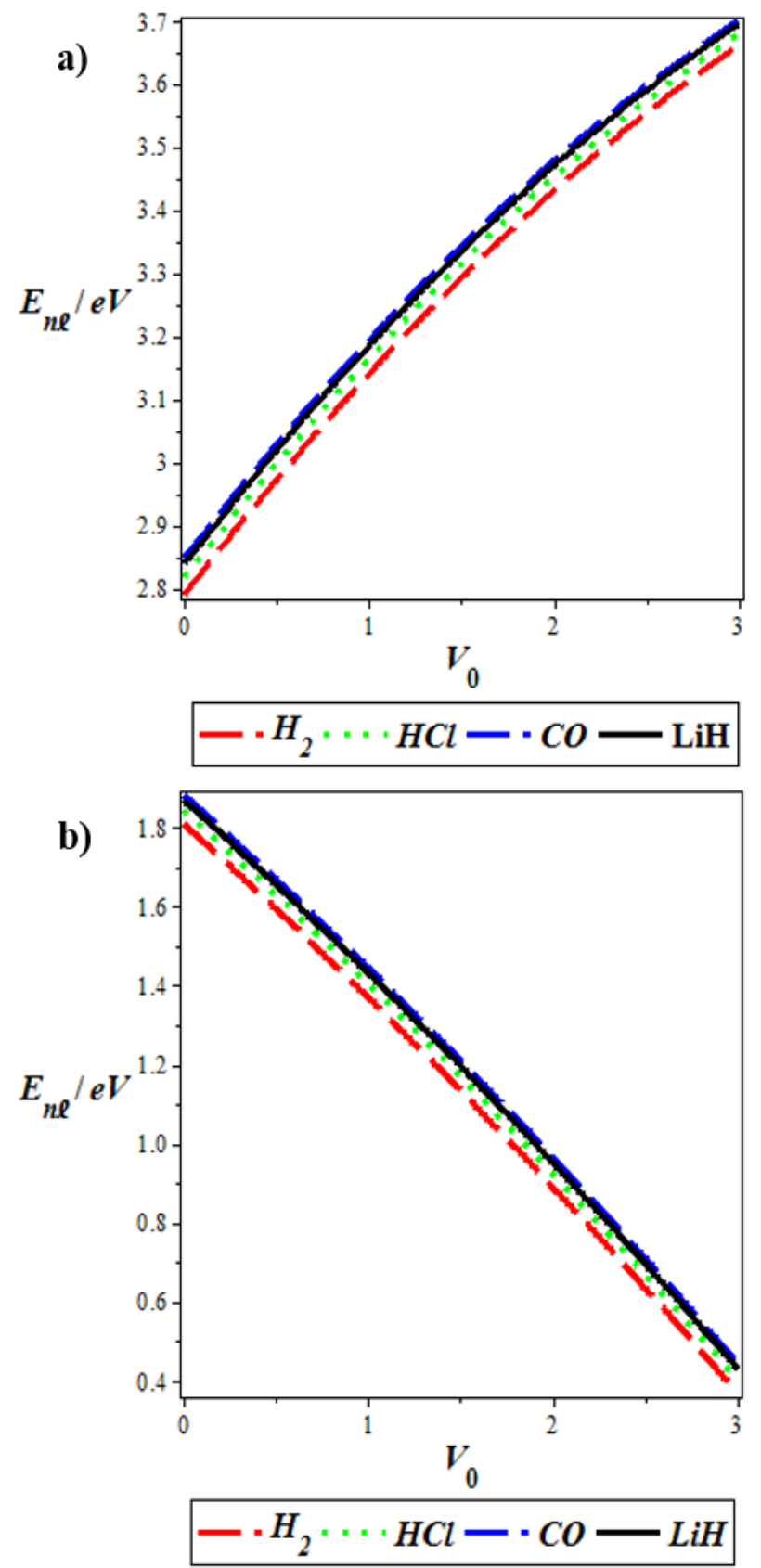

Figure 2. (a) The variation of the energy spectrum for various values of $n$ as a function of the parameter $V_{0}$ for $q=1$. (b) The variation of the energy spectrum for various values of $n$ as a function of the parameter $V_{0}$ for $\mathrm{q}=-1 \mathrm{~h}=1, \mu=1, \mathrm{a}=2, \mathrm{~b}=1, \mathrm{c}=-3, \mathrm{~V}_{1}=$ 2 and $\alpha=0.05$.
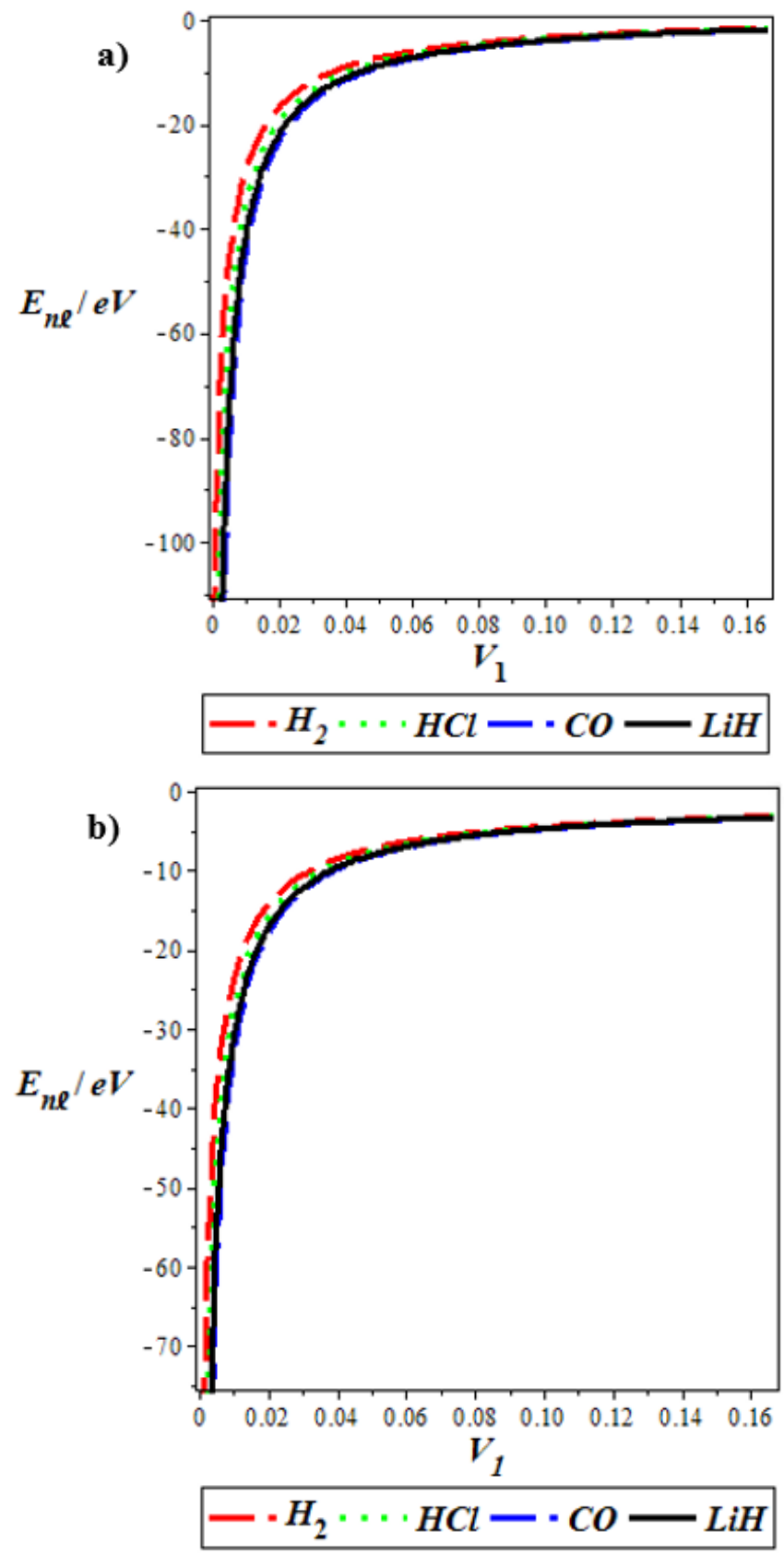

Figure 3. (a)The variation of the energy spectrum for various values of $n$ as a function of the parameter $V_{l}$ for $q=1$. (b) The variation of the energy spectrum for various values of $n$ as a function of the parameter $V_{l}$ for $\mathrm{q}=-1 . \mathrm{a}=2, \mathrm{~b}=1, \mathrm{c}=-3$ and $\mathrm{V}_{0}=3$. 

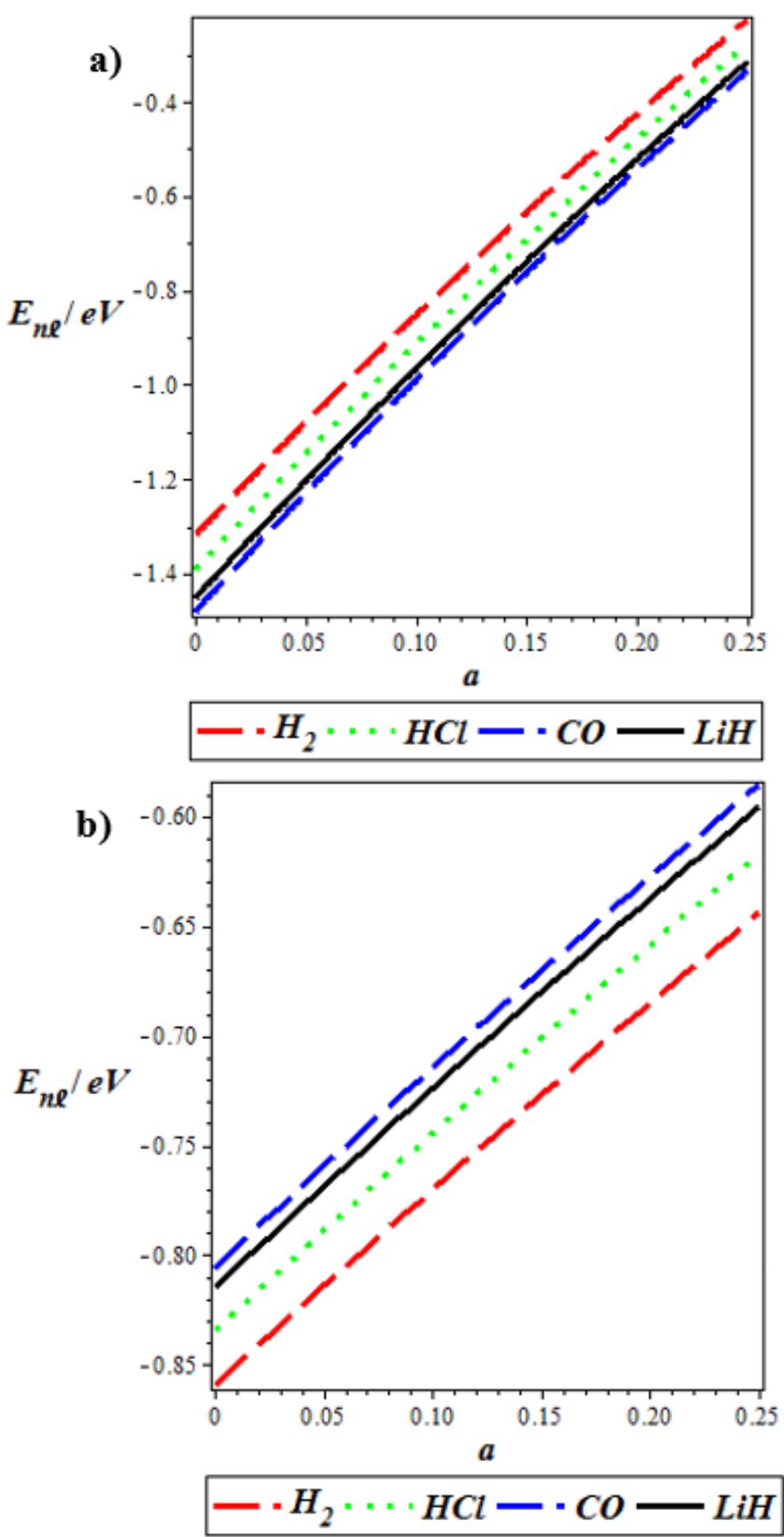

Figure 4. (a) The variation of the energy spectrum for various values of $n$ as a function of the parameter $a$ for $q=1$. (b) The variation of the energy spectrum for various values of $n$ as a function of the parameter $a$ for $\mathrm{q}=-1 . \mathrm{b}=1, \mathrm{c}=-3, \mathrm{~V}_{0}=3$ and $V_{1}=2$.
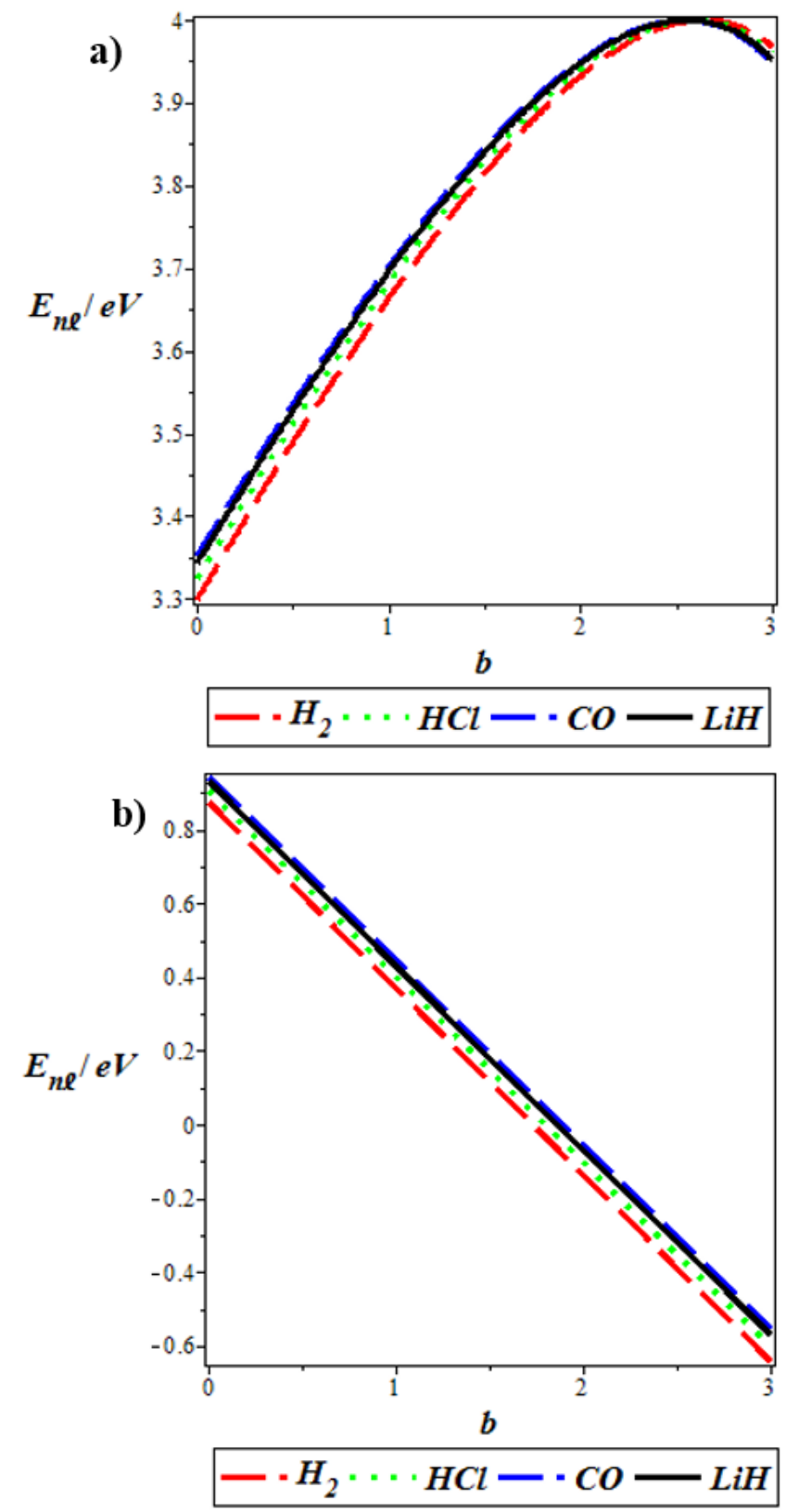

Figure 5. (a) The variation of the energy spectrum for various values of $n$ as a function of the parameter $b$ for $\mathrm{q}=1$. (b) The variation of the energy spectrum for various values of $n$ as a function of the parameter $b$ for $\mathrm{q}=-1 . \mathrm{a}=2, \mathrm{c}=-3, \mathrm{~V}_{0}=3$ and $V_{1}=2$. 

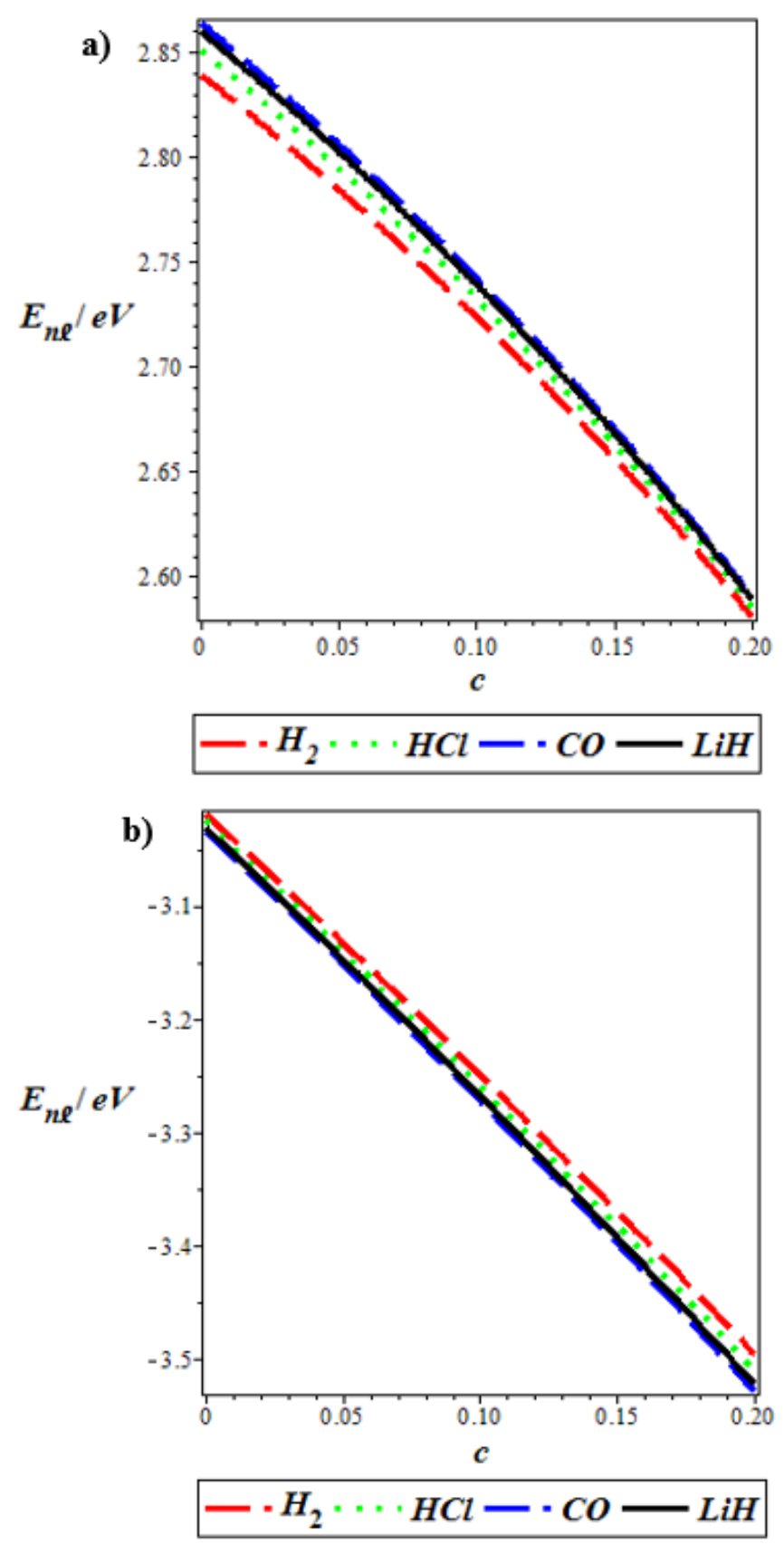

Figure 6. (a) The variation of the energy spectrum for various values of $n$ as a function of the parameter $c$ for $q=1$. (b) The variation of the energy spectrum for various values of $n$ as a function of the parameter $c$ for $\mathrm{q}=-1 . \mathrm{a}=2, \mathrm{~b}=1, \mathrm{~V}_{0}=3$ and $V_{1}=2$.
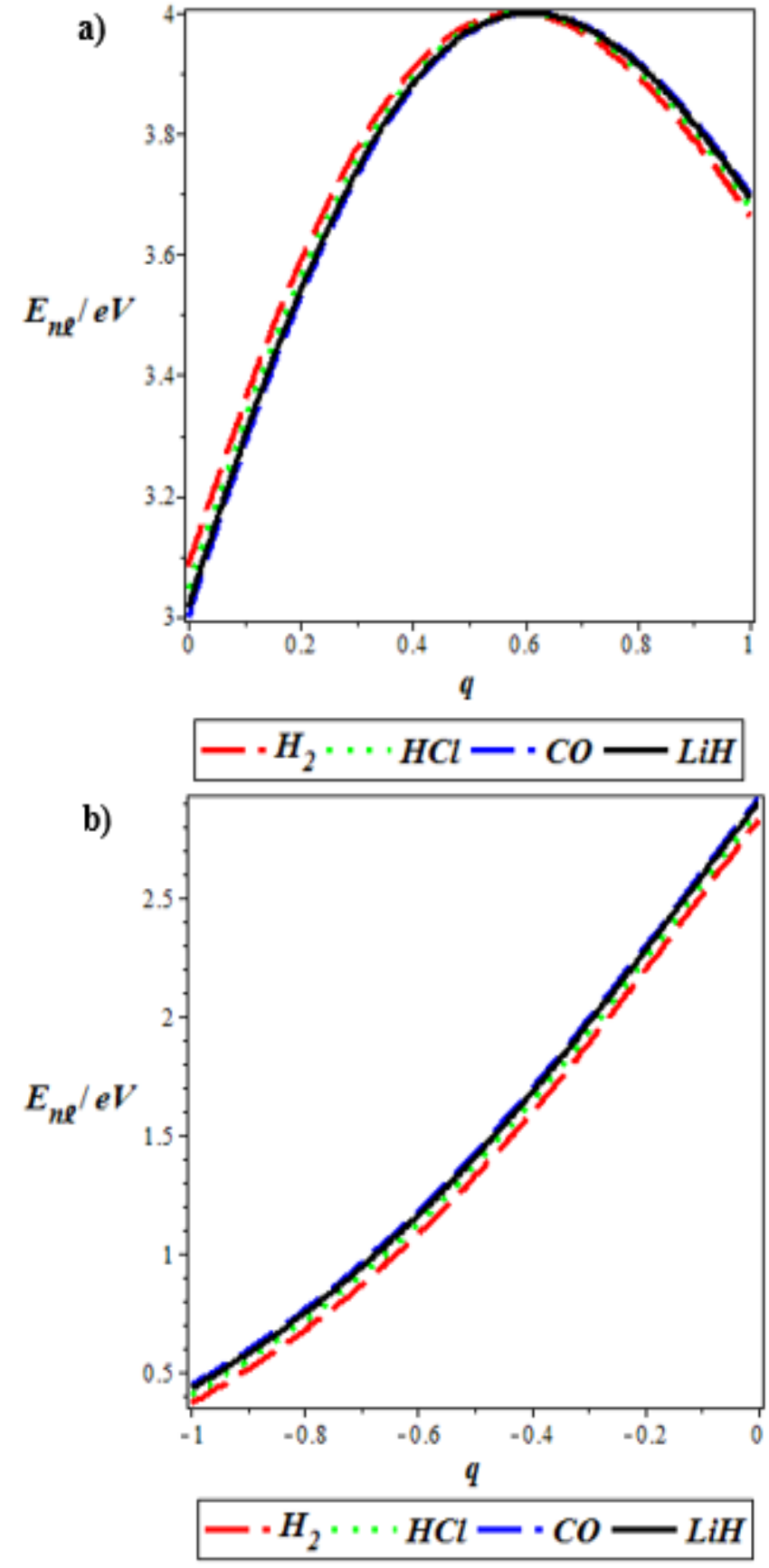

Figure 7. (a) The variation of the energy spectrum for various values of $n$ as a function of the parameter $-q$. (b) The variation of the energy spectrum for various values of $n$ as a function of the parameter $+q . \mathrm{a}=2$, $\mathrm{b}=1, \mathrm{c}=-3, \mathrm{~V}_{1}=2$ and $\mathrm{V}_{0}=3$. 


\section{Conclusion}

In this work, the bound state solutions of the Schrödinger equation were studied with the $q$ deformed Hulthen-quadratic exponential-type potential using parametric NU method. The Greene and Aldrich approximation scheme was used to deal with the centrifugal term; the energy eigenvalues and the corresponding eigenfunctions were obtained and some special cases of the potential were also discussed. The energy spectrum is applied to study four selected diatomic molecules, $\mathrm{H}_{2}, \mathrm{HCl}, \mathrm{CO}$ and $\mathrm{LiH}$. The effect of the deformation parameters and other potential parameters on the energy of the system were graphically and numerically analyzed. The results are in excellent agreement with literature. Finally, the results can find many applications in quantum mechanical systems, atomic and molecular physics.

\section{Authors' contribution}

Conceptualization: Obogo, U. P.; Ubi, O. E.

Data curation: Ikot, A. N.; Edet, C. O.

Formal Analysis: Edet, C. O.

Funding acquisition: Not applicable.

Investigation: Obogo, U. P.; Ubi, O. E.; Edet, C. O.; Ikot, A. N.

Methodology: Edet, C. O.

Project administration: Obogo, U. P.; Ubi, O. E.; Edet, C. O.; Ikot, A. N.

Resources: Edet, C. O.; Ikot, A. N.

Software: Edet, C. O.

Supervision: Obogo, U. P.; Edet, C. O.; Ikot, A. N.

Validation: Edet, C. O.; Ikot, A. N.

Visualization: Edet, C. O.; Ikot, A. N.

Writing - original draft: Obogo, U. P.; Ubi, O. E.

Writing - review \& editing: Edet, C. O.; Ikot, A. N.

\section{Data availability statement}

All data sets were generated or analyzed in the current study.

\section{Funding}

Not applicable

\section{Acknowledgments}

We thank the anonymous referees for the positive enlightening comments and suggestions, which have greatly helped us in making improvements to this paper. In addition, Collins Okon Edet acknowledges eJDS (ICTP).

\section{References}

Agboola, D. The Hulthén potential in D-dimensions. Phys. Scr. 2009, 80 (6), 065304. https://doi.org/10.1088/0031$8949 / 80 / 06 / 065304$

Bayrak, O.; Kocak, G.; Boztosun, I. Any $l$-state solutions of the Hulthén potential by the asymptotic iteration method. $J$. Phys. A: Math. Gen. 2006, 39 (37), 11521. https://doi.org/10.1088/0305-4470/39/37/012

Ciftci, H.; Hall, R. L.; Saad, N. Asymptotic iteration method for eigenvalue problems. J. Phys. A: Math. Gen. 2003, 36 (47), 11807. https://doi.org/10.1088/0305-4470/36/47/008

Ciftci, H.; Hall, R. L.; Saad, N. Perturbation theory in a framework of iteration methods. Phys. Lett. A 2005, 340 (56), 388-396. https://doi.org/10.1016/j.physleta.2005.04.030

Dong, S.-H. Factorization method in quantum mechanics; Springer, 2007

Durmus, A.; Yasuk, F. Relativistic and nonrelativistic solutions for diatomic molecules in the presence of double ring-shaped Kratzer potential. J. Chem. Phys 2007, 126, 074108. https://doi.org/10.1063/1.2566432

Ebomwonyi, O.; Onate, C. A.; Onyeaju, M. C.; Ikot, A. N. Any $\ell$-states solutions of the Schrödinger equation interacting with Hellmann-generalized Morse potential model. Karbala Intl. J. Mod. Sci. 2017, 3 (1), 59-68. https://doi.org/10.1016/j.kijoms.2017.03.001

Edet, C. O.; Okoi, P. O. Any $l$-state solutions of the Schrödinger equation for $q$-deformed Hulthen plus generalized inverse quadratic Yukawa potential in arbitrary dimensions. Rev. Mex. Fis. 2019, 65 (4), 333-344. https://doi.org/10.31349/RevMexFis.65.333

Edet, C. O.; Okoi, P. O.; Chima S. O. Analytic solutions of the Schrödinger equation with non-central generalized inverse quadratic Yukawa potential. Rev. Bras. Ensino Fís. 2020a, 42, e20190083. https://doi.org/10.1590/1806-9126rbef-2019-0083

Edet, C. O., Okorie, U. S., Ngiangia, A. T., Ikot, A. N. Bound state solutions of the Schrodinger equation for the modified Kratzer potential plus screened Coulomb potential. Indian J. Phys. 2020b, 94, 425-433. https://doi.org/10.1007/s12648-019-01477-9

Edet, C. O.; Amadi, P. O.; Okorie, U. S.; Tas, A.; Ikot, A. N.; Rampho, G. Solutions of Schrodinger equation and thermal properties of generalized trigonometric PoschlTeller potential. Rev. Mex. Fis. 2020c, 66 (6). https://doi.org/10.31349/RevMexFis.66.824 
Edet, C. O.; Okorie, K. O.; Louis, H.; Nzeata-Ibe, N. A. Any $l$-state solutions of the Schrodinger equation interacting with Hellmann-Kratzer potential model. Indian J. Phys. 2020d, 94, 243-251. https://doi.org/10.1007/s12648-019-01467-x

Edet, C. O.; Okorie, U. S.; Osobonye, G.; Ikot, A. N.; Rampho, G. J.; Sever, R. Thermal properties of Deng-FanEckart potential model using Poisson summation approach. J. Math. Chem. 2020e, 58, 989-1013. https://doi.org/10.1007/s10910-020-01107-4

Edet C. O.; Amadi, P. O.; Onyeaju, M. C.; Okorie, U. S.; Sever, R.; Rampho, G. J.; Abdullah, H. Y.; Salih, I. H.; Ikot, A. N. Thermal Properties and Magnetic Susceptibility of Hellmann Potential in Aharonov-Bohm (AB) Flux and Magnetic Fields at Zero and Finite Temperatures. J. Low Temp. Phys. 2021a, 202, 83-105. https://doi.org/10.1007/s10909-020-02533-z

Edet, C. O.; Ikot, A. N.; Onyeaju, M. C.; Okorie, U. S.; Rampho, G. J.; Lekala, M. L.; Kaya, S. Thermo-magnetic properties of the screened Kratzer potential with spatially varying mass under the influence of Aharanov-Bohm(AB) and position-dependent magnetic fields. Physica E. 2021b, 131, 114710. https://doi.org/10.1016/j.physe.2021.114710

Falaye, B. J. Any $\ell$-state solutions of the Eckart potential via asymptotic iteration method. Cent. Eur. Phys. 2012, 10 (4), 960-965. https://doi.org/10.2478/s11534-012-0047-6

Falaye, B. J.; Oyewumi, K. J.; Ikhdair, S. M.; Hamzavi, M. Eigensolution techniques, their applications and Fisher's information entropy of the Tietz-Wei diatomic molecular model. Phys. Scr. 2014, 89 (11), 115204. https://doi.org/10.1088/0031-8949/89/11/115204

Falaye, B. J.; Ikhdair, S. M.; Hamzavi, M. Energy States of Some Diatomaic Molecules: The Exact Quantisation Rule Approach. Zeitschrift für Naturforschung A 2015a, 70 (2), 85-90. https://doi.org/10.1515/zna-2014-0232

Falaye, B. J.; Ikhdair, S. M.; Hamzavi, M. Formula Method for Bound State Problems. Few-Body Syst. 2015b, 56, 6378. https://doi.org/10.1007/s00601-014-0937-9

Greene, R. L.; Aldrich, C. Variational wave functions for a screened Coulomb potential. Phys. Rev. A 1976, 14 (6), 2363. https://doi.org/10.1103/PhysRevA.14.2363

Greiner W. Relativistic Quantum Mechanics: Wave equations; Springer, 2000. https://doi.org/10.1007/978-3662-04275-5

Gu, X.-Y.; Dong, S.-H. Energy spectrum of the ManningRosen potential including centrifugal term solved by exact and proper quantization rules. J. Math. Chem. 2011, 49, 2053. https://doi.org/10.1007/s10910-011-9877-5

Hall, R. L.; Saad, N.; Sen, K. D. Exact normalized eigenfunctions for general deformed Hulthén potentials. $J$. $\begin{array}{llll}\text { Math. } & \text { Phys. } & \text { 2018, } & \text { 59, }\end{array}$ https://doi.org/10.1063/1.5043484

Ikhdair, S. M. An improved approximation scheme for the centrifugal term and the Hulthén potential. Eur. Phys. J. A 2009, 39, 307-314. https://doi.org/10.1140/epja/i200810715-2

Ikhdair, S. M.; Sever, R. Approximate Eigenvalue and Eigenfunction Solutions for the Generalized Hulthén Potential with any Angular Momentum. J. Math. Chem. 2007, 42, 461-471. https://doi.org/10.1007/s10910-0069115-8

Ikot, A. N.; Awoga, O. A.; Hassanabadi, H.; Maghsoodi, E. Analytical approximate solution of Schrödinger equation in $D$ dimensions with quadratic exponential-type potential for arbitrary l-State. Commun. Theor. Phys. 2014, 61 (4), 457. https://doi.org/10.1088/0253-6102/61/4/09

Ikot A. N.; Edet, C. O.; Amadi, P. O.; Okorie, U. S.; Rampho, G. J.; Abdullah, H. Y. Thermodynamic properties of Aharanov-Bohm (AB) and magnetic fields with screened Kratzer potential. Eur. Phys. J. D 2020a, 74, 159. https://doi.org/10.1140/epjd/e2020-10084-9

Ikot, A. N.; Okorie U. S.; Osobonye G.; Amadi P. O.; Edet C. O.; Sithole M. J.; Rampho G. J.; Sever R. Superstatistics of Schrödinger equation with pseudo-harmonic potential in external magnetic and Aharanov-Bohm fields. Heliyon 2020b, 60 (4), e03738. https://doi.org/10.1016/j.heliyon.2020.e03738

Ita, B. I.; Louis, H.; Akakuru, O. U.; Nzeata-Ibe, N. A.; Ikeuba A. I.; Magu, T. O.; Amos, P. I.; Edet, C. O. Approximate Solution to the Schrödinger Equation with Manning- Rosen plus a Class of Yukawa Potential via WKBJ Approximation Method. Bulg. J. Phys. 2018, 45, 323-333.

Jia, C.-S.; Liu, J.-Y.; Wang, P.-Q. A new approximation scheme for the centrifugal term and the Hulthén potential. Phys. Lett. A 2008, 372 (27-28), 4779-4782. https://doi.org/10.1016/j.physleta.2008.05.030

Landau, L. D.; Lifshitz E. M. Quantum Mechanics: Nonrelativistic Theory; Pergamon, 1977.

Louis, H.; Iserom, I. B.; Akakuru, O. U.; Nzeata-Ibe, N. A.; Ikeuba, A. I.; Magu, T. O.; Amos, P. I.; Collins, E. O. $l$-state Solutions of the Relativistic and Non-Relativistic Wave Equations for Modified Hylleraas-Hulthen Potential Using the Nikiforov-Uvarov Quantum Formalism. Orient. J. Phys. Sciences 2018a, 3 (1).

Louis H.; Ita B. I.; Magu T. O.; Akakuru U. O.; Nzeata-Ibe, N.; Ikeuba I. A.; Amos I. P.; Edet C. O. Solutions to the Dirac Equation for Manning-Rosen Plus Shifted Deng-Fan Potential and Coulomb-Like Tensor Interaction Using Nikiforov-Uvarov Method. Int. J. Chem. 2018b, 10 (3), 99106. https://doi.org/10.5539/ijc.v10n3p99 
Ma, Z.-Q.; Xu, B.-W. Quantum correction in exact quantization rules. EPL (Europhysics Letters) 2005, 69 (5), 685. https://doi.org/10.1209/epl/i2004-10418-8

Nikiforov, A. F.; Uvarov, V. B. Special Functions of Mathematical Physics; Birkhäuser Verlag Basel, 1988.

Okoi, P. O.; Edet C. O.; Magu T. O. Relativistic treatment of the Hellmann-generalized Morse potential. Rev. Mex. Fis. 2020, $66 \quad$ (1), 1-13. https://doi.org/10.31349/RevMexFis.66.1

Okorie, U. S.; Ikot, A. N.; Onyeaju, M. C.; Chukwuocha, E. O. A study of thermodynamic properties of quadratic exponential-type potential in D-dimensions. Rev. Mex. Fis. 2018, 64 (6), 608-614. https://doi.org/10.31349/RevMexFis.64.608

Okorie U. S.; Ikot A. N.; Edet C. O.; Rampho, G. J.; Server, R.; Akpan I. O. Solutions of the Klein Gordon equation with generalized hyperbolic potential in D-dimensions. J. Phys. Commun. 2019, 3 (9), 095015. https://doi.org/10.1088/23996528/ab42c6

Okorie U. S.; Edet C. O.; Ikot A. N.; Rampho G. J.; Sever R., Thermodynamic functions for diatomic molecules with modified Kratzer plus screened Coulomb potential. Indian J. Phys. 2020, 95, 411-421. https://doi.org/10.1007/s12648019-01670-w

Onate, C. A.; Onyeaju, M. C.; Ikot, A. N.; Ojonubah J. O. Eigen solutions, Shannon entropy and fisher information under the Eckart Manning Rosen potential model. J. Korean Phys. Soc. 2017, 30, 339-347. https://doi.org/10.3938/jkps.70.339

Onate, C. A.; Adebimpe, O.; Adebesin, B. O; Lukman, A. F. Information-theoretic measure of the hyperbolical exponential-type potential. Turk. J. Phys. 2018a, 42 (4), 402-414. https://doi.org/10.3906/fiz-1802-40

Onate, C. A.; Adebimpe, O.; Lukman, A. F.; Adama, I. J.; Okoro, J. O.; Davids, E. O. Approximate eigensolutions of the attractive potential via parametric Nikiforov-Uvarov method. Heliyon 2018b, 4 (11), e00977. https://doi.org/10.1016/j.heliyon.2018.e00977

Onate, C. A.; Ikot, A. N.; Onyeaju, M. C.; Ebomwonyi, O.; Idiodi, J. O. A. Effect of dissociation energy on Shannon and Rényi entropies. Karbala Int. J. Mod. Sci. 2018c, 4 (1), 134142. https://doi.org/10.1016/j.kijoms.2017.12.004

Qiang, W.-C.; Dong, S.-H. Arbitrary $l$-state solutions of the rotating Morse potential through the exact quantization rule method. Phys. Lett. A 2007, 363 (3), 169-176. https://doi.org/10.1016/j.physleta.2006.10.091

Rampho, G. J.; Ikot A. N.; Edet C. O.; Okorie U. S. Energy spectra and thermal properties of diatomic molecules in the presence of magnetic and $\mathrm{AB}$ fields with improved Kratzer potential. Mol. Phys. 2021, 119 (5), e1821922. https://doi.org/10.1080/00268976.2020.1821922

\section{Schiff L. I. Quantum Mechanics; McGraw Hill, 1995.}

Tezcan, C.; Sever, R. A general approach for the exact solution of the Schrödinger equation. Intl. J. Theor. Phys. 2009, 48, 337-350. https://doi.org/10.1007/s10773-0089806-y 